\title{
TOPOLOGICAL COARSE SHAPE GROUPS OF COMPACT METRIC SPACES
}

\author{
Nikola Koceić Bilan and ZdRavko ČUKa
}

Dedicated to professor Sibe Mardešić, with respect, homage and gratitude.

\begin{abstract}
The shape theory and, relatively new, coarse shape theory are very useful in studying of topological spaces, as well as of the corresponding algebraic invariants, especially, shape and coarse shape groups.

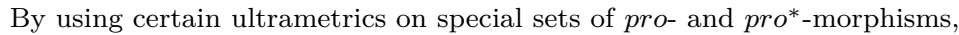
we topologize those groups when they refer to compact metric spaces and we get topological groups. In the shape case, they are isomorphic to recently constructed topological shape homotopy groups, while in the coarse shape case we get the coarse shape invariants, denoted by $\check{\pi}_{k}^{* d^{*}}\left(X, x_{0}\right)$. We have proven some important properties of $\check{\pi}_{k}^{* d^{*}}\left(X, x_{0}\right)$ and provided few interesting examples.
\end{abstract}

\section{INTRODUCTION}

The shape and coarse shape groups are important invariants of shape and coarse shape theory, respectively. Recently, certain topology has been constructed on shape groups which turns them into topological groups (naturally called topological shape homotopy groups, see [5]) and it has been shown that they are new shape invariants, with ability to differ shape of some pairs of topological spaces which regular shape groups cannot differ. It is important to emphasize that topology shape (homotopy) groups of compact metric spaces are metrizable.

N. Uglešić has constructed ultrametrics $d$ and $d^{*}$ on pro- $\mathcal{C}(\boldsymbol{X}, \boldsymbol{Y})$ and pro* $-\mathcal{C}(\boldsymbol{X}, \boldsymbol{Y})$, respectively, for any category $\mathcal{C}$ and any inverse systems $\boldsymbol{X}, \boldsymbol{Y}$ in $\mathcal{C}$, where $\boldsymbol{Y}$ is cofinite (each element of the corresponding index set has at most finitely many predecessors). Ultrametric spaces which we get by applying those ultrametrics are denoted by $\left(\boldsymbol{Y}^{\mathbf{X}}, d\right)$ and $\left(\boldsymbol{Y}^{\mathbf{X} *}, d^{*}\right)$. Also, he

2010 Mathematics Subject Classification. 55P55, 55Q05, 55N99.

Key words and phrases. Inverse system, pro-category, pro*-category, expansion, shape, coarse shape, homotopy pro-group, shape group, coarse shape group, topological shape homotopy group, coarse shape path connectedness. 
showed that those topological spaces can be quite different if codomain is changed up to isomorphism. However, this problem can be solved if one restricts this consideration to inverse sequences only, precisely to subcategories tow- $\mathcal{C}$, tow $^{*}-\mathcal{C}$.

So, clearly, the sets of shape and coarse shape morphisms between topological spaces $X$ and $Y$, with the extra condition that $X$ and $Y$ admits sequential HPol-expansions (compact metric spaces make a special case), can be turned into ultrametric spaces, independently of choosing specific sequential expansions.

The previous construction can be applied to sets of pointed shape and coarse shape morphisms, as well. Therefore, by restricting the domain to the pointed sphere, the shape groups and coarse shape groups (of compact metric spaces or, more general, of spaces admitting sequential expansions) can be topologized. We will prove that, in the both cases, topological groups are obtained, which will be denoted by $\check{\pi}_{k}^{d}\left(X, x_{0}\right)$ and $\check{\pi}_{k}^{* d^{*}}\left(X, x_{0}\right)$.

In the case of shape groups, these topological groups are isomorphic to topological shape groups ([5]) (according to this, no new name will be needed). Thus we obtain an explicit (complete) metric for those spaces. Nevertheless, in the case of coarse shape, we get a benefit of the new topological-algebraic invariant, which will be named by a topological coarse shape group. A certain topology (similar to the topology given in Lemma 4.5) which induces a structure of a topological group on the coarse shape group is already considered in $([6])$.

The shape and coarse shape case is going to be mutually related by showing that a topological shape group can be considered as a closed topological subgroup of the corresponding topological coarse shape group. Some interesting properties of topological coarse shape groups will be considered.

At the end, we will discuss few examples, one of which will be a non stable space whose topological coarse shape group is nontrivial and discrete.

\section{Preliminaries}

Let us give a brief review of main notions and categories important for this paper. Let $\mathcal{C}$ be any category. Categories $i n v-\mathcal{C}$ and $i n v^{*}-\mathcal{C}$ are defined as follows.

Objects of inv-C are all the inverse systems $\boldsymbol{X}=\left(X_{\lambda}, p_{\lambda \lambda^{\prime}}, \Lambda\right)$ in $\mathcal{C}$ and inv-C $(\boldsymbol{X}, \boldsymbol{Y})$ is the set of all pairs $\left(f, f_{\mu}\right): \boldsymbol{X} \rightarrow \boldsymbol{Y}=\left(Y_{\mu}, q_{\mu \mu^{\prime}}, M\right)$ which consists of an index function $f: M \rightarrow \Lambda$ and of morphisms $f_{\mu}: X_{f(\mu)} \rightarrow Y_{\mu}$ in $\mathcal{C}$, for every $\mu \in M$, satisfying following condition: for every $\mu \leq \mu^{\prime}$ in $M$ there is a $\lambda \in \Lambda, \lambda \geq f(\mu), f\left(\mu^{\prime}\right)$ such that $f_{\mu} p_{f(\mu) \lambda}=q_{\mu \mu^{\prime}} f_{\mu^{\prime}} p_{f\left(\mu^{\prime}\right) \lambda}$ holds.

Objects of $i n v^{*}-\mathcal{C}$ are also all the inverse systems $\boldsymbol{X}$ in $\mathcal{C}$ and $i n v^{*}-\mathcal{C}(\boldsymbol{X}, \boldsymbol{Y})$ is the set of all pairs $\left(f, f_{\mu}^{n}\right): \boldsymbol{X} \rightarrow \boldsymbol{Y}$ (called $*$-morphisms) which consists of an index function $f: M \rightarrow \Lambda$ and of sequences of morphisms $f_{\mu}^{n}: X_{f(\mu)} \rightarrow Y_{\mu}$ 
in $\mathcal{C}, n \in \mathbb{N}$, for every $\mu \in M$, such that following condition is satisfied: for every $\mu \leq \mu^{\prime}$ in $M$ there is a $\lambda \in \Lambda, \lambda \geq f(\mu), f\left(\mu^{\prime}\right)$ and there is an $n \in \mathbb{N}$, so that, for every $n^{\prime} \geq n$, relation $f_{\mu}^{n^{\prime}} p_{f(\mu) \lambda}=q_{\mu \mu^{\prime}} f_{\mu^{\prime}}^{n^{\prime}} p_{f\left(\mu^{\prime}\right) \lambda}$ holds.

The composition of (*-)morphisms $\left(f, f_{\mu}\right): \boldsymbol{X} \rightarrow \boldsymbol{Y}$ and $\left(g, g_{\nu}\right)$ : $\boldsymbol{Y} \rightarrow \boldsymbol{Z}=\left(Z_{\nu}, r_{\nu \nu^{\prime}}, N\right) \quad\left(\left(f, f_{\mu}^{n}\right): \boldsymbol{X} \rightarrow \boldsymbol{Y}\right.$ and $\left.\left(g, g_{\nu}^{n}\right): \boldsymbol{Y} \rightarrow \boldsymbol{Z}\right)$ is the (*-)morphism $\left(f \circ g, g_{\nu} \circ f_{g(\nu)}\right): \boldsymbol{X} \rightarrow \boldsymbol{Z}\left(\left(f \circ g, g_{\nu}^{n} \circ f_{g(\nu)}^{n}\right): \boldsymbol{X} \rightarrow \boldsymbol{Z}\right)$ and the identity (*-)morphism on $\boldsymbol{X}$ is $\left(1_{\Lambda}, 1_{x_{\lambda}}\right): \boldsymbol{X} \rightarrow \boldsymbol{X}\left(\left(1_{\Lambda}, 1_{x_{\lambda}}^{n}=1_{x_{\lambda}}\right): \boldsymbol{X} \rightarrow \boldsymbol{X}\right)$, where $1_{\Lambda}: \Lambda \rightarrow \Lambda$ is the identity index function and $1_{x_{\lambda}}$ are identity morphisms in $\mathcal{C}$.

Two morphisms $\left(f, f_{\mu}\right),\left(f^{\prime}, f_{\mu}^{\prime}\right): \boldsymbol{X} \rightarrow \boldsymbol{Y}$ of $i n v-\mathcal{C}$ are said to be equivalent, denoted by $\left(f, f_{\mu}\right) \sim\left(f^{\prime}, f_{\mu}^{\prime}\right)$, if every $\mu \in M$ admits $\lambda \in \Lambda$, $\lambda \geq f(\mu), f^{\prime}(\mu)$ such that $f_{\mu} p_{f(\mu) \lambda}=f_{\mu}^{\prime} p_{f^{\prime}(\mu) \lambda}$ holds, and two *-morphisms $\left(f, f_{\mu}^{n}\right),\left(f^{\prime}, f_{\mu}^{\prime n}\right): \boldsymbol{X} \rightarrow \boldsymbol{Y}$ of $i n v^{*}-\mathcal{C}$ are said to be equivalent, denoted by $\left(f, f_{\mu}^{n}\right) \sim\left(f^{\prime}, f_{\mu}^{\prime n}\right)$, if every $\mu \in M$ admits $\lambda \in \Lambda, \lambda \geq f(\mu), f^{\prime}(\mu)$ and $n \in \mathbb{N}$, such that, for every $n^{\prime} \geq n$, relation $f_{\mu}^{n^{\prime}} p_{f(\mu) \lambda}=f_{\mu}^{\prime n^{\prime}} p_{f^{\prime}(\mu) \lambda}$ is satisfied.

Previously defined relations are equivalence relations which are compatible with composition in $i n v-\mathcal{C}$ and $i n v^{*}-\mathcal{C}$, respectively. So, there exists corresponding quotient categories $i n v-\mathcal{C} / \sim \equiv$ pro- $\mathcal{C}$ and $i n v^{*}-\mathcal{C} / \sim \equiv$ pro $^{*}-\mathcal{C}$. Morphisms $\left[\left(f, f_{\mu}\right)\right],\left[\left(f, f_{\mu}^{n}\right)\right]$ of pro-C, pro $^{*}-\mathcal{C}$, respectively, are denoted by $\boldsymbol{f}$ and $\boldsymbol{f}^{*}$. Especially, in this paper we will be interested into two full subcategories tow- $\mathcal{C} \subset$ pro- $\mathcal{C}$, tow $^{*}-\mathcal{C} \subset$ pro $^{*}-\mathcal{C}$, whose objects are all inverse sequences in $\mathcal{C}$.

Also, there is a functor $\boldsymbol{J}:$ pro- $\mathcal{C} \rightarrow$ pro $^{*}-\mathcal{C}$, defined as follows: $\boldsymbol{J}(\boldsymbol{X})=\boldsymbol{X}$, for every $\boldsymbol{X} \in O b($ pro- $\mathcal{C})$ and $\boldsymbol{J}\left(\boldsymbol{f}=\left[\left(f, f_{\mu}\right)\right]\right)=\boldsymbol{f}^{*}=\left[\left(f, f_{\mu}^{n}\right)\right]$, for every $\boldsymbol{f} \in \operatorname{pro-\mathcal {C}}(X, Y)$, where $f_{\mu}^{n}=f_{\mu}$ for every $\mu \in M$ and $n \in \mathbb{N}\left(\boldsymbol{f}^{*}\right.$ is said to be induced by $\boldsymbol{f})$. Whereas functor $\boldsymbol{J}$ is faithful and fixes objects, we can consider pro- $\mathcal{C}$ as a subcategory of pro* $^{*}-\mathcal{C}$.

Now, let $\mathcal{D}$ be a pro-reflective and full subcategory of $\mathcal{C}$, which means that every $X \in O b(\mathcal{C})$ admits a $\mathcal{D}$-expansion (see more in [7]). Let $\boldsymbol{p}: X \rightarrow$ $\boldsymbol{X}, \boldsymbol{p}^{\prime}: X \rightarrow \boldsymbol{X}^{\prime}$ be $\mathcal{D}$-expansions of $X \in O b(\mathcal{C})$ and $\boldsymbol{q}: Y \rightarrow \boldsymbol{Y}, \boldsymbol{q}^{\prime}: Y \rightarrow \boldsymbol{Y}^{\prime}$ be $\mathcal{D}$-expansions of $Y \in O b(\mathcal{C})$, and let $\boldsymbol{i}: \boldsymbol{X} \rightarrow \boldsymbol{X}^{\prime}, \boldsymbol{j}: \boldsymbol{Y} \rightarrow \boldsymbol{Y}^{\prime}$ be canonical (by definition of $\mathcal{D}$-expansion) isomorphisms in pro- $\mathcal{D}$. We say that two promorphisms $\boldsymbol{f}: \boldsymbol{X} \rightarrow \boldsymbol{Y}, \boldsymbol{f}^{\prime}: \boldsymbol{X}^{\prime} \rightarrow \boldsymbol{Y}^{\prime}$ are pro- $\mathcal{D}$ equivalent if $\boldsymbol{j} \circ \boldsymbol{f}=\boldsymbol{f}^{\prime} \circ \boldsymbol{i}$ and two pro*-D morphisms $\boldsymbol{f}^{*}: \boldsymbol{X} \rightarrow \boldsymbol{Y}, \boldsymbol{f}^{\prime *}: \boldsymbol{X}^{\prime} \rightarrow \boldsymbol{Y}^{\prime}$ are said to be pro* $^{*}$ $\mathcal{D}$ equivalent provided $\boldsymbol{J}(\boldsymbol{j}) \circ \boldsymbol{f}^{*}=\boldsymbol{f}^{\prime *} \circ \boldsymbol{J}(\boldsymbol{i})$ holds. These two relations are equivalence relations on the appropriate subclass of $\operatorname{Mor}($ pro- $\mathcal{D})$, Mor $\left(\right.$ pro*- $^{*}$ $\mathcal{D})$, respectively, and they are compatible with composition whenever it is defined. Equivalence classes of $\boldsymbol{f}$ and $\boldsymbol{f}^{*}$ are denoted by $\langle\boldsymbol{f}\rangle$ and $\left\langle\boldsymbol{f}^{*}\right\rangle$.

At this step we can introduce the (abstract) shape category $S h_{(\mathcal{C}, \mathcal{D})}$ and (abstract) coarse shape category $S h_{(\mathcal{C}, \mathcal{D})}^{*}$ for a pair $(\mathcal{C}, \mathcal{D})$ (see more in [4] and [7]). Objects of these two categories are all objects of $\mathcal{C}$ and morphisms $F \in S h_{(\mathcal{C}, \mathcal{D})}(X, Y), F^{*} \in S h_{(\mathcal{C}, \mathcal{D})}^{*}(X, Y)$ (naturally called shape and coarse 
shape morphisms) are pro- $\mathcal{D}$ and pro $^{*}-\mathcal{D}$ equivalence classes of morphisms in pro- $\mathcal{D}(\boldsymbol{X}, \boldsymbol{Y})$ and pro*$^{*}-\mathcal{D}(\boldsymbol{X}, \boldsymbol{Y})$, respectively, with respect to any choice of a pair of $\mathcal{D}$-expansions $\boldsymbol{p}: X \rightarrow \boldsymbol{X}, \boldsymbol{q}: Y \rightarrow \boldsymbol{Y}$. The composition in the both categories is defined via the representatives and the identities on an object $X$ are $\left\langle\mathbf{1}_{\boldsymbol{X}}\right\rangle,\left\langle\mathbf{1}_{\boldsymbol{X}}^{*}\right\rangle$, where $\mathbf{1}_{\boldsymbol{X}}, \mathbf{1}_{\boldsymbol{X}}^{*}: \boldsymbol{X} \rightarrow \boldsymbol{X}$ are identity morphisms in pro- $\mathcal{D}$, pro $^{*}-\mathcal{D}$, respectively, and $\boldsymbol{p}: X \rightarrow \boldsymbol{X}$ is any $\mathcal{D}$-expansion of $X$.

Here are some main functorial relations. Let $\boldsymbol{p}: X \rightarrow \boldsymbol{X}, \boldsymbol{q}: Y \rightarrow \boldsymbol{Y}$ be $\mathcal{D}$-expansions of $X$ and $Y$, respectively. Then, for every morphism $f: X \rightarrow Y$ in $\mathcal{C}$ there is a unique $\boldsymbol{f}: \boldsymbol{X} \rightarrow \boldsymbol{Y}$ in pro- $\mathcal{D}$ such that $\boldsymbol{q} \circ[(f)]=\boldsymbol{f} \circ \boldsymbol{p}$ in pro-C. Shape functor $S: \mathcal{C} \rightarrow S h_{(\mathcal{C}, \mathcal{D})}$ is defined as $S(X)=X, S(f)=\langle\boldsymbol{f}\rangle$ and coarse shape functor $S^{*}: \mathcal{C} \rightarrow S h_{(\mathcal{C}, \mathcal{D})}^{*}$ as $S^{*}(X)=X, S^{*}(f)=\langle\boldsymbol{J}(\boldsymbol{f})\rangle$. It can be easily seen that $S^{*}=J_{(\mathcal{C}, \mathcal{D})} \circ S$, where $J_{(\mathcal{C}, \mathcal{D})}$ is the functor from shape to coarse shape category, induced by the previously mentioned functor $\boldsymbol{J}$ restricted to pro- $\mathcal{D}$. Since $J_{(\mathcal{C}, \mathcal{D})}$ is a faithful functor the shape category can be considered as a subcategory of the coarse shape category.

At last, let us recall the well known facts that HPol (the homotopy category of all polyhedra) is a pro-reflective full subcategory of HTop (the homotopy category of all topological spaces). Also, the analogous result holds for pointed topological spaces "case" $\mathrm{HPol}_{0} \subset H T o p_{0}$. In this paper we will mainly restrict our consideration to $S h_{0} \equiv S h_{\left(H T o p_{0}, H P o l_{0}\right)}$ (the pointed shape category) and $S h_{0}^{*} \equiv S h_{\left(H T o p_{0}, H P_{0} l_{0}\right)}^{*}$ (the pointed coarse shape category) because main objects of our research are shape and coarse shape groups of topological spaces, although most of constructions and conclusions can be applicable to a more general case.

\section{UltrametrizATION OF SHAPE AND COARSE SHAPE GROUP}

First, we give a quick reminder about definition of shape and coarse shape group of (pointed) topological space $\left(X, x_{0}\right)$. For $k \in \mathbb{N}$ let $\left(S^{k}, s_{0}\right)$ be the pointed $k$-dimensional sphere and let $\boldsymbol{p}=\left[\left(p_{\lambda}\right)\right]:\left(X, x_{0}\right) \rightarrow\left(\left(X_{\lambda}, x_{\lambda}\right), p_{\lambda \lambda^{\prime}}, \Lambda\right)$ be any $H \mathrm{Pol}_{0}$-expansion of $\left(X, x_{0}\right)$. The $k$-dimensional shape group of $\left(X, x_{0}\right)$, denoted by $\check{\pi}_{k}\left(X, x_{0}\right)$, is the set $S h_{0}\left(\left(S^{k}, s_{0}\right),\left(X, x_{0}\right)\right)$, with the binary operation defined as follows:

$$
A+B=\left\langle\left[\left(a_{\lambda}\right)\right]\right\rangle+\left\langle\left[\left(b_{\lambda}\right)\right]\right\rangle=\left\langle\left[\left(a_{\lambda}+b_{\lambda}\right)\right]\right\rangle .
$$

Similarly, the $k$-dimensional coarse shape group of $\left(X, x_{0}\right)$, denoted by $\check{\pi}_{k}^{*}\left(X, x_{0}\right)$, is the set $S h_{0}^{*}\left(\left(S^{k}, s_{0}\right),\left(X, x_{0}\right)\right)$, with the binary operation

$$
A^{*}+B^{*}=\left\langle\left[\left(a_{\lambda}^{n}\right)\right]\right\rangle+\left\langle\left[\left(b_{\lambda}^{n}\right)\right]\right\rangle=\left\langle\left[\left(a_{\lambda}^{n}+b_{\lambda}^{n}\right)\right]\right\rangle .
$$

Notice that for an $\mathrm{HPol}_{0}$-expansion of sphere we may choose "identity". These two structures are groups (for $k \geq 2$ Abelian groups) with adding operation independent of choosing $\mathrm{HPol}_{0}$-expansion $\boldsymbol{p}$. For a (coarse) shape morphism $\left(A^{*}\right) A,\left(\left(A^{*}\right)^{-1}=\left\langle\left[\left(\left(a_{\lambda}^{n}\right)^{-1}\right)\right]\right\rangle \in \check{\pi}_{k}^{*}\left(X, x_{0}\right)\right)(A)^{-1}=\left\langle\left[\left(\left(a_{\lambda}\right)^{-1}\right)\right]\right\rangle \in$ 
$\check{\pi}_{k}\left(X, x_{0}\right)$ is the inverse element, where $\left(\left(a_{\lambda}^{n}\right)^{-1}\right)\left(a_{\lambda}\right)^{-1}:\left(S^{k}, s_{0}\right) \rightarrow\left(X_{\lambda}, x_{\lambda}\right)$ is the inverse for $\left(a_{\lambda}^{n}\right) a_{\lambda}$ in $\pi_{k}\left(X_{\lambda}, x_{\lambda}\right)$, for all $n \in \mathbb{N}$ and $\lambda \in \Lambda$. From now on, we will consider only topological spaces admitting sequential polyhedral expansions (compact metric spaces make a special case ). Let $\left(X, x_{0}\right)$ be any such pointed space and let $\boldsymbol{p}=\left[\left(p_{i}\right)\right]:\left(X, x_{0}\right) \rightarrow\left(\boldsymbol{X}, \boldsymbol{x}_{0}\right)=\left(\left(X_{i}, x_{i}\right), p_{i i+1}, \mathbb{N}\right)$ be its polyhedral expansion. Let $\left(\boldsymbol{S}^{k}, \boldsymbol{s}_{0}\right)$ denotes a rudimentary inverse system having only one object $\left(S^{k}, s_{0}\right)$ and let

$$
\begin{aligned}
\left(\boldsymbol{X}, \boldsymbol{x}_{0}\right)^{\left(\boldsymbol{S}^{k}, \boldsymbol{s}_{0}\right)} & :=\text { pro-HPol}_{0}\left(\left(\boldsymbol{S}^{k}, \boldsymbol{s}_{0}\right),\left(\boldsymbol{X}, \boldsymbol{x}_{0}\right)\right), \\
\left(\boldsymbol{X}, \boldsymbol{x}_{0}\right)^{\left(\boldsymbol{S}^{k}, \boldsymbol{s}_{0}\right) *}: & =\text { pro }^{*}-\operatorname{HPol}_{0}\left(\left(\boldsymbol{S}^{k}, \boldsymbol{s}_{0}\right),\left(\boldsymbol{X}, \boldsymbol{x}_{0}\right)\right) .
\end{aligned}
$$

By [8] and [9], $\left(\left(\boldsymbol{X}, \boldsymbol{x}_{0}\right)^{\left(\boldsymbol{S}^{k}, \boldsymbol{s}_{0}\right)}, d\right)$ and $\left(\left(\boldsymbol{X}, \boldsymbol{x}_{0}\right)^{\left(\boldsymbol{S}^{k}, \boldsymbol{s}_{0}\right) *}, d^{*}\right)$ are complete ultrametric spaces, where

$$
\begin{array}{r}
d:\left(\boldsymbol{X}, \boldsymbol{x}_{0}\right)^{\left(\boldsymbol{S}^{k}, \boldsymbol{s}_{0}\right)} \times\left(\boldsymbol{X}, \boldsymbol{x}_{0}\right)^{\left(\boldsymbol{S}^{k}, \boldsymbol{s}_{0}\right)} \rightarrow \mathbb{R}, \\
d\left(\left[\left(a_{i}\right)\right],\left[\left(b_{i}\right)\right]\right)=\left\{\begin{array}{cc}
\inf \left\{\frac{1}{m+1} \mid\right. & \left.a_{m}=b_{m}, m \in \mathbb{N}\right\} \\
1, & \text { otherwise }
\end{array}\right\}
\end{array}
$$

and

$$
d^{*}:\left(\boldsymbol{X}, \boldsymbol{x}_{0}\right)^{\left(\mathbf{S}^{k}, \mathbf{s}_{0}\right) *} \times\left(\boldsymbol{X}, \boldsymbol{x}_{0}\right)^{\left(\boldsymbol{S}^{k}, \boldsymbol{s}_{0}\right) *} \rightarrow \mathbb{R}
$$

$d^{*}\left(\left[\left(a_{i}^{n}\right)\right],\left[\left(b_{i}^{n}\right)\right]\right)=\left\{\begin{array}{c}\inf \left\{\frac{1}{m+1} \mid a_{m}^{n}=b_{m}^{n} \text { for almost all } n \in \mathbb{N} ; m \in \mathbb{N}\right\} \\ 1,\end{array}\right\}$.

If we take another sequential polyhedral expansion of $\left(X, x_{0}\right)$ then the obtained ultrametric spaces are mutually homeomorphic. The corresponding homeomorphisms are induced by natural isomorphisms between expansions (Theorem 6. in [8] and Theorem 6. in [9]). Thus, the natural bijections $\left(\mathbf{X}, \mathbf{x}_{0}\right)^{\left(\mathbf{S}^{k}, \mathbf{s}_{0}\right)} \approx \check{\pi}_{k}\left(X, x_{0}\right)$ and $\left(\mathbf{X}, \mathbf{x}_{0}\right)^{\left(\mathbf{S}^{k}, \mathbf{s}_{0}\right) *} \approx \check{\pi}_{k}^{*}\left(X, x_{0}\right)$ can be used to topologize the shape and coarse shape group, independently of specifying sequential expansion. The obtained structures will be denoted by $\check{\pi}_{k}^{d}\left(X, x_{0}\right)$ and $\check{\pi}_{k}^{* d^{*}}\left(X, x_{0}\right)$, respectively.

Proposition 3.1. Let $\left\langle\left[\left(a_{i}\right)\right]\right\rangle,\left\langle\left[\left(b_{i}\right)\right]\right\rangle \in \check{\pi}_{k}\left(X, x_{0}\right) \quad\left(\left\langle\left[\left(a_{i}^{n}\right)\right]\right\rangle,\left\langle\left[\left(b_{i}^{n}\right)\right]\right\rangle \in\right.$ $\left.\check{\pi}_{k}^{*}\left(X, x_{0}\right)\right)$. If $d\left(\left[\left(a_{i}\right)\right],\left\langle\left[\left(b_{i}\right)\right]\right\rangle\right)=\frac{1}{m}\left(d^{*}\left(\left[\left(a_{i}^{n}\right)\right],\left[\left(b_{i}^{n}\right)\right]\right)=\frac{1}{m}\right), m \in \mathbb{N}, m>1$, then $a_{i}=b_{i}\left(a_{i}^{n}=b_{i}^{n}\right.$ for almost all $\left.n \in \mathbb{N}\right)$, for every $i=1, \ldots, m-1$.

Proof. Since $a_{m}=b_{m}\left(a_{m}^{n}=b_{m}^{n}\right.$ for almost all $\left.n \in \mathbb{N}\right)$ implies $p_{i m} a_{m}=$ $p_{i m} b_{m}\left(p_{i m} a_{m}^{n}=p_{i m} b_{m}^{n}\right.$ for almost all $\left.n \in \mathbb{N}\right)$ the statement follows by the definition of $(*-)$ morphism.

THEOREM 3.2. $\check{\pi}_{k}^{d}\left(X, x_{0}\right)$ and $\check{\pi}_{k}^{* d^{*}}\left(X, x_{0}\right)$ are topological groups, for every $k \in \mathbb{N}$. 
Proof. We will prove the coarse shape case only. The proof of the first one is analogous and even easier. Let $k \in \mathbb{N}$. Let $A^{*}=\left\langle\left[\left(a_{i}^{n}\right)\right]\right\rangle$ and $B^{*}=\left\langle\left[\left(b_{i}^{n}\right)\right]\right\rangle$ be any elements of $\check{\pi}_{k}^{* d^{*}}\left(X, x_{0}\right)$, and let $m \in \mathbb{N}$. By properties of the homotopy classes addition on each coordinate it is easy to check that for any $\left(C^{*}, D^{*}\right) \in B_{d^{*}}\left(A^{*}, 1 / m\right) \times B_{d^{*}}\left(B^{*}, 1 / m\right)$ we have $C^{*}+D^{*} \in B_{d^{*}}\left(A^{*}+B^{*}, 1 / m\right)$, so continuity of the addition on the $\check{\pi}_{k}^{* d^{*}}\left(X, x_{0}\right)$ follows. Now take any $A^{*}=\left\langle\left[\left(a_{i}^{n}\right)\right]\right\rangle$ and any $m \in \mathbb{N}$. Consider the inverse $\left(A^{*}\right)^{-1}=\left\langle\left[\left(\left(a_{i}^{n}\right)^{-1}\right)\right]\right\rangle$. If we choose any $B^{*}=\left\langle\left[\left(b_{i}^{n}\right)\right]\right\rangle \in B_{d^{*}}\left(A^{*}, 1 / m\right)$, by properties of taking inverses in the $k$-dimensional homotopy groups of polyhedra $\left(X_{i}, x_{i}\right)$, it follows directly that $\left(B^{*}\right)^{-1} \in B_{d^{*}}\left(\left(A^{*}\right)^{-1}, 1 / m\right)$. Now we infer that the operation of taking inverses is continuous on $\check{\pi}_{k}^{* d^{*}}\left(X, x_{0}\right)$. Hence $\check{\pi}_{k}^{* d^{*}}\left(X, x_{0}\right)$ is a topological group.

The topological shape group $\check{\pi}_{k}^{t o p}\left(X, x_{0}\right)$, constructed in [5], has for elements of its topological basis the sets

$$
V_{\lambda_{0}}^{A=\left\langle\left[\left(a_{\lambda}\right)\right]\right\rangle}=\left\{B=\left\langle\left[\left(b_{\lambda}\right)\right]\right\rangle \in \check{\pi}_{k}\left(X, x_{0}\right) \mid b_{\lambda_{0}}=a_{\lambda_{0}}\right\},
$$

for all $A \in \check{\pi}_{k}\left(X, x_{0}\right)$ and $\lambda_{0} \in \Lambda$, where

$$
\mathbf{p}=\left[\left(p_{\lambda}\right)\right]:\left(X, x_{0}\right) \rightarrow\left(\left(X_{\lambda}, x_{\lambda}\right), p_{\lambda \lambda^{\prime}}, \Lambda\right)
$$

is any $\mathrm{HPol}_{0}$-expansion of $\left(X, x_{0}\right)$. By restricting our consideration only to compact metric spaces $\left(X, x_{0}\right)$ and by using any sequential polyhedral expansion $\mathbf{p}=\left[\left(p_{i}\right)\right]:\left(X, x_{0}\right) \rightarrow\left(\left(X_{i}, x_{i}\right), p_{i i+1}, \mathbb{N}\right)$ it can be easily proved that the natural bijection $\check{\pi}_{k}^{d}\left(X, x_{0}\right) \rightarrow \check{\pi}_{k}^{t o p}\left(X, x_{0}\right)$ induces an isomorphism of topological groups, for every $k \in \mathbb{N}$. Indeed, for any base element $V_{m_{0}}^{A}$ of the topological shape group $\check{\pi}_{k}^{t o p}\left(X, x_{0}\right)$, by using Proposition 3.1, one can prove that the ball $B\left(A=\left[\left(a_{i}\right)\right], \frac{1}{m_{0}}\right)$ of the metric space $\check{\pi}_{k}^{d}\left(X, x_{0}\right)$ is contained in $V_{m_{0}}^{A}$. Also, any ball $B\left(A=\left[\left(a_{i}\right)\right], r\right)$ contains $V_{m}^{A}, m \geq \frac{1}{r}$. This leads us to few conclusions.

Corollary 3.3. $\check{\pi}_{k}^{t o p}\left(X, x_{0}\right)$ is a metrizable space, for any $k \in \mathbb{N}$ and any space $\left(X, x_{0}\right)$ which admits a sequential HPol $H_{0}$-expansion. Furthermore, $\check{\pi}_{k}^{t o p}\left(X, x_{0}\right)$ is homeomorphic to the complete ultrametric space $\check{\pi}_{k}^{d}\left(X, x_{0}\right)$.

COROLlarY 3.4. Let $S h_{0 c M}$ be the full subcategory of $S h_{0}$ restricted to the all compact metric spaces and let TopGrp ${ }_{M_{c}}$ be the full subcategory of TopGrp (the category of topological groups) restricted to the all metrizable topological groups admitting metrization by a complete metric. There is a functor $\check{\pi}_{k}^{d}: S h_{0 c M} \rightarrow$ TopGrp $M_{c}$ which associates $\check{\pi}_{k}^{d}\left(X, x_{0}\right)$ with any $\left(X, x_{0}\right) \in$ $O b\left(S h_{0 c M}\right)$ and continuous homomorphism $\check{\pi}_{k}^{d}(F): \check{\pi}_{k}^{d}\left(X, x_{0}\right) \rightarrow \check{\pi}_{k}^{d}\left(Y, y_{0}\right)$, $\check{\pi}_{k}^{d}(F)(A)=F A$, with any $F \in S_{0 c M}\left(\left(X, x_{0}\right),\left(Y, y_{0}\right)\right)$.

Corollary 3.5. For every shape path connected space $X$, the topological group $\check{\pi}_{k}^{d}\left(X, x_{0}\right)$ does not depend on the choice of a base point $x_{0}$. 
COROllary 3.6. $\check{\pi}_{k}^{d}\left(X, x_{0}\right)$ can be considered as the inverse limit of $\left(\left(\pi_{k}^{d}\left(X_{i}, x_{i}\right), \pi_{k}^{d}\left(S\left(p_{i i+1}\right)\right), \mathbb{N}\right)\right.$ taken in TopGrp.

\section{Topological COARSE Shape Groups of COMPACT METRiC SPACES}

Now let us research several main properties of the topological group $\check{\pi}_{k}^{* d^{*}}\left(X, x_{0}\right)$, which will be naturally called (sequential) topological coarse shape group of the space $\left(X, x_{0}\right)$ (recall that it is only defined for spaces admitting sequential polyhedral expansion). Since all described constructions are rather natural, we are able to define an appropriate functor.

Proposition 4.1. Let $S h_{0_{\mathbb{N}}^{*}}$ be the full subcategory of $S h_{0}^{*}$ restricted to

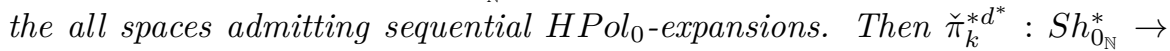
TopGrp $M_{M_{c}}$, which associates $\check{\pi}_{k}^{* d^{*}}\left(X, x_{0}\right)$ with any $\left(X, x_{0}\right) \in S h_{0_{\mathbb{N}}^{*}}^{*}$ and $\check{\pi}_{k}^{* d^{*}}\left(F^{*}\right): \check{\pi}_{k}^{* d^{*}}\left(X, x_{0}\right) \rightarrow \check{\pi}_{k}^{* d^{*}}\left(Y, y_{0}\right), \check{\pi}_{k}^{* d^{*}}\left(F^{*}\right)\left(A^{*}\right)=F^{*} A^{*}$, with any $F^{*} \in$ $S h_{0_{\mathbb{N}}}^{*}\left(\left(X, x_{0}\right),\left(Y, y_{0}\right)\right)$, is a well defined functor.

PROOF. This proposition follows by Theorem 3.2, properties of the coarse shape functor defined in [1] and Corollary 3.3 of [9].

We are going to call the functor $\check{\pi}_{k}^{* d^{*}}: S h_{0_{\mathbb{N}}}^{*} \rightarrow \operatorname{TopGrp} M_{c}$ (sequential) topological coarse shape group functor. By composing it with the forgetful functor $\operatorname{TopGrp}_{M_{c}} \rightarrow \operatorname{Grp}$, we get the coarse shape group functor restricted to $S h_{0_{\mathbb{N}}}^{*}$. Also, it is known that shape groups can be embedded into coarse shape groups by the homomorphism induced by the functor $J_{\left(H T o p_{0}, H \text { Pol }_{0}\right)}$ (see [1]). By combining this fact with Theorem 2.3. of [9], we can deduce the next proposition.

Proposition 4.2. The function $j: \check{\pi}_{k}^{d}\left(X, x_{0}\right) \rightarrow \check{\pi}_{k}^{* d^{*}}\left(X, x_{0}\right), j(A)=$ $J(A)$ is an injective homomorphism and a closed topological embedding.

Therefore $\check{\pi}_{k}^{d}\left(X, x_{0}\right)$ can be considered as a closed topological subgroup of $\check{\pi}_{k}^{* d^{*}}\left(X, x_{0}\right)$. Also, it would be interesting if one can somehow relate a topological coarse shape group of a subspace to the topological coarse shape group of the whole space. We will give a complete answer when a subspace is a retract, which will be an analogy of the "shape" case, already considered in [5], with a slight improvement.

Definition 4.3. Let $X$ be a topological space and let $X_{0}$ be its subspace. We say that $X_{0}$ is normally embedded in $X$ if for every normal covering $\mathcal{U}_{0}$ of $X_{0}$ there exists a normal covering $\mathcal{U}$ of $X$ such that $\left.\mathcal{U}\right|_{X_{0}}=\left(U \cap X_{0}: U \in \mathcal{U}\right)$ refines $\mathcal{U}_{0}$.

Proposition 4.4. Let $X$ be a topological space which admits a sequential $\mathrm{HPol}_{0}$-expansion and let $X_{0}$ be a subspace normally embedded in $X$

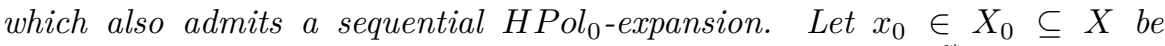
any point, $i:\left(X_{0}, x_{0}\right) \hookrightarrow\left(X, x_{0}\right)$ the inclusion and $i_{\#}^{*}:=\check{\pi}_{k}^{* d^{*}}\left(S^{*}\left(\bar{H}_{0}(i)\right)\right)$ : 
$\check{\pi}_{k}^{* d^{*}}\left(X_{0}, x_{0}\right) \rightarrow \check{\pi}_{k}^{* d^{*}}\left(X, x_{0}\right)$, where $H_{0}$ is the pointed homotopy functor. Then $i_{\#}^{*}\left(\check{\pi}_{k}^{* d^{*}}\left(X_{0}, x_{0}\right)\right)$ is closed in $\check{\pi}_{k}^{* d^{*}}\left(X, x_{0}\right)$.

Proof. Because $X_{0}$ is normally embedded in $X$, by Theorem 6.11. of [7] there is a morphism $\boldsymbol{p}=\left[\left(p_{\lambda}\right)\right]:\left(X, X_{0}\right) \rightarrow\left(\boldsymbol{X}, \boldsymbol{X}_{0}\right)=\left(\left(X_{\lambda}, X_{0 \lambda}\right), p_{\lambda \lambda^{\prime}}, \Lambda\right)$ in pro-Top ${ }^{2}$, where $\left(\boldsymbol{X}, \boldsymbol{X}_{0}\right)$ is an inverse system in Pol $^{2}$ (category of polyhedral pairs $)$, such that $\left.\boldsymbol{p}\right|_{X}=\left[\left(p_{\lambda}\right)\right]: X \rightarrow\left(X_{\lambda}, p_{\lambda \lambda^{\prime}}, \Lambda\right)$ and $\left.\boldsymbol{p}\right|_{X_{0}}=\left[\left(\left.p_{\lambda}\right|_{X_{0}}\right)\right]$ : $X_{0} \rightarrow\left(X_{0 \lambda},\left.p_{\lambda \lambda^{\prime}}\right|_{X_{0 \lambda^{\prime}}}, \Lambda\right)$ are polyhedral resolutions of spaces $X$ and $X_{0}$, respectively (more on polyhedral resolutions in [7]). Further, by Lemma 2.3 of [3], if we put $x_{\lambda}=p_{\lambda}\left(x_{0}\right)=\left.p_{\lambda}\right|_{X_{0}}\left(x_{0}\right)$ and use the functor $\boldsymbol{H}_{0}$ : proTop $_{0} \rightarrow$ pro-HTop 0 which is induced by $H_{0}$, we have that

$$
\begin{aligned}
\boldsymbol{H}_{0}\left(\left.\boldsymbol{p}\right|_{X}\right) & =\left[\left(\left[p_{\lambda}\right]\right)\right]:\left(X, x_{0}\right) \rightarrow\left(\mathbf{X}, \mathbf{x}_{0}\right)=\left(\left(X_{\lambda}, x_{\lambda}\right),\left[p_{\lambda \lambda^{\prime}}\right], \Lambda\right), \\
\boldsymbol{H}_{0}\left(\left.\boldsymbol{p}\right|_{X_{0}}\right) & =\left[\left(\left[\left.p_{\lambda}\right|_{X_{0}}\right]\right)\right]:\left(X_{0}, x_{0}\right) \rightarrow\left(\mathbf{X}_{0}, \mathbf{x}_{0}\right)=\left(\left(X_{0 \lambda}, x_{\lambda}\right),\left[\left.p_{\lambda \lambda^{\prime}}\right|_{X_{0 \lambda^{\prime}}}\right], \Lambda\right)
\end{aligned}
$$

are $\mathrm{HPol}_{0}$-expansions of spaces $\left(X, x_{0}\right)$ and $\left(X_{0}, x_{0}\right)$, respectively.

Now, let $\boldsymbol{q}=\left[\left(\left[q_{i}\right]\right)\right]:\left(X, x_{0}\right) \rightarrow\left(\boldsymbol{Y}, \mathbf{y}_{0}\right)=\left(\left(Y_{i}, y_{i}\right),\left[q_{i i+1}\right], \mathbb{N}\right), \boldsymbol{r}=$ $\left[\left(\left[r_{i}\right]\right)\right]:\left(X_{0}, x_{0}\right) \rightarrow\left(\boldsymbol{Z}, \boldsymbol{z}_{0}\right)=\left(\left(Z_{i}, z_{i}\right),\left[r_{i i+1}\right], \mathbb{N}\right)$ be any sequential $\mathrm{HPol}_{0^{-}}$ expansions of $\left(X, x_{0}\right),\left(X_{0}, x_{0}\right)$, respectively. Then, because of natural bijective relations

$$
\begin{aligned}
\check{\pi}_{k}^{*}\left(X, x_{0}\right) & \approx \operatorname{pro}^{*}-\operatorname{PPol}_{0}\left(\left(\boldsymbol{S}^{k}, \boldsymbol{s}_{0}\right),\left(\boldsymbol{Y}, \boldsymbol{y}_{0}\right)\right) \\
& \approx \operatorname{pro}^{*}-\operatorname{PPol}_{0}\left(\left(\boldsymbol{S}^{k}, \mathbf{s}_{0}\right),\left(\boldsymbol{X}, \boldsymbol{x}_{0}\right)\right) \\
\check{\pi}_{k}^{*}\left(X_{0}, x_{0}\right) & \approx \text { pro }^{*}-\operatorname{PPol}_{0}\left(\left(\boldsymbol{S}^{k}, \boldsymbol{s}_{0}\right),\left(\boldsymbol{Z}, \boldsymbol{z}_{0}\right)\right) \\
& \approx \text { pro }^{*}-\operatorname{PPol}_{0}\left(\left(\boldsymbol{S}^{k}, \boldsymbol{s}_{0}\right),\left(\boldsymbol{X}_{0}, \boldsymbol{x}_{0}\right)\right)
\end{aligned}
$$

by using Lemma 4.5 below, in order to prove that $i_{\#}^{*}\left(\check{\pi}_{k}^{* d^{*}}\left(X_{0}, x_{0}\right)\right)$ is closed in $\check{\pi}_{k}^{* d^{*}}\left(X, x_{0}\right)$, it is enough to prove that $k\left(\right.$ pro $\left.^{*}-H \operatorname{Pol}_{0}\left(\left(\boldsymbol{S}^{k}, \boldsymbol{s}_{0}\right),\left(\boldsymbol{X}_{0}, \boldsymbol{x}_{0}\right)\right)\right)$ is closed in pro* $^{*}-\operatorname{PPol}_{0}\left(\left(\boldsymbol{S}^{k}, \boldsymbol{s}_{0}\right),\left(\boldsymbol{X}, \boldsymbol{x}_{0}\right)\right)$, where the topology on pro* $^{*}-\operatorname{HPol}_{0}\left(\left(\boldsymbol{S}^{k}, \boldsymbol{s}_{0}\right),\left(\boldsymbol{X}, \boldsymbol{x}_{0}\right)\right)$ is defined as in Lemma 4.5 , while $k$ is the function

$$
\begin{gathered}
k: \operatorname{pro}^{*}-H \operatorname{Pol}_{0}\left(\left(\mathbf{S}^{k}, \mathbf{s}_{0}\right),\left(\boldsymbol{X}_{0}, \boldsymbol{x}_{0}\right)\right) \rightarrow \operatorname{pro}^{*}-H \operatorname{Pol}_{0}\left(\left(\boldsymbol{S}^{k}, \boldsymbol{s}_{0}\right),\left(\boldsymbol{X}, \boldsymbol{x}_{0}\right)\right), \\
k\left(\left[\left(\left[a_{\lambda}^{n}\right]\right)\right]\right)=\left[\left(j,\left[j_{\lambda}^{n}\right]\right)\right] \circ\left[\left(\left[a_{\lambda}^{n}\right]\right)\right],
\end{gathered}
$$

where $\left[\left(j,\left[j_{\lambda}^{n}\right]\right)\right]$ represents $S^{*}\left(H_{0}(i)\right)$ for given and fixed $H \mathrm{Pol}_{0}$-expansions $\boldsymbol{H}_{0}\left(\left.\boldsymbol{p}\right|_{X_{0}}\right)$ and $\boldsymbol{H}_{0}\left(\left.\boldsymbol{p}\right|_{X}\right)$.

Now, by using the obvious fact that $\boldsymbol{H}_{0}\left(\left.\boldsymbol{p}\right|_{X}\right) \circ[([i])]=\left[\left(1_{\lambda},\left[i_{\lambda}\right]\right)\right] \circ$ $\boldsymbol{H}_{0}\left(\left.\boldsymbol{p}\right|_{X_{0}}\right)$ in pro-HTop prere $\left[\left(1_{\Lambda},\left[i_{\lambda}\right]\right)\right]:\left(\boldsymbol{X}_{0}, \boldsymbol{x}_{0}\right) \rightarrow\left(\boldsymbol{X}, \boldsymbol{x}_{0}\right), 1_{\Lambda}: \Lambda \rightarrow \Lambda$ is the identity, $i_{\lambda}:\left(X_{0 \lambda}, x_{\lambda}\right) \hookrightarrow\left(X_{\lambda}, x_{\lambda}\right)$ are inclusions, for every $\lambda \in \Lambda$, we conclude that $j=1_{\Lambda},\left[j_{\lambda}^{n}\right]=\left[i_{\lambda}\right]$, for every $\lambda \in \Lambda, n \in \mathbb{N}$.

We will complete this proof by showing that

$$
K=\text { pro }^{*}-H \operatorname{Pol}_{0}\left(\left(\mathbf{S}^{k}, \mathbf{s}_{0}\right),\left(\boldsymbol{X}, \boldsymbol{x}_{0}\right)\right) \backslash k\left(\operatorname{pro}^{*}-\operatorname{HPol}_{0}\left(\left(\boldsymbol{S}^{k}, \boldsymbol{s}_{0}\right),\left(\boldsymbol{X}_{0}, \boldsymbol{x}_{0}\right)\right)\right)
$$


is open in pro* $^{*}-\operatorname{HPol}_{0}\left(\left(\boldsymbol{S}^{k}, \boldsymbol{s}_{0}\right),\left(\boldsymbol{X}, \boldsymbol{x}_{0}\right)\right)$. Let $\boldsymbol{a}=\left[\left(\left[a_{\lambda}^{n}\right]\right)\right] \in K$. Then there exists $\lambda_{0} \in \Lambda$ such that for every $n_{0} \in \mathbb{N}$ there exists $n^{\prime} \in \mathbb{N}, n^{\prime} \geq n_{0}$, such that $\left[a_{\lambda_{0}}^{n^{\prime}}\right] \neq\left[i_{\lambda_{0}} f\right]$, for every continuous mapping $f:\left(S^{k}, s_{0}\right) \rightarrow\left(X_{0 \lambda_{0}}, x_{\lambda_{0}}\right)$. Namely, if it is not so, then for every $\lambda \in \Lambda$ we can choose $n(\lambda) \in \mathbb{N}$ such that for every $n^{\prime} \geq n(\lambda)$ we can find the continuous mapping $\bar{a}_{0 \lambda}^{n^{\prime}}:\left(S^{k}, s_{0}\right) \rightarrow$ $\left(X_{0 \lambda}, x_{\lambda}\right)$ such that $\left[a_{\lambda}^{n^{\prime}}\right]=\left[j_{\lambda}^{n^{\prime}} \bar{a}_{0 \lambda}^{n^{\prime}}\right]$. It allows us to define mappings $a_{0 \lambda}^{n}$ : $\left(S^{k}, s_{0}\right) \rightarrow\left(X_{0 \lambda}, x_{\lambda}\right)$

$$
a_{0 \lambda}^{n}=\left\{\begin{array}{ll}
\text { const, } & n<n(\lambda) \\
\bar{a}_{0 \lambda}^{n}, & n \geq n(\lambda)
\end{array}\right\}
$$

for every $\lambda \in \Lambda, n \in \mathbb{N}$, and to infer that $\boldsymbol{a}_{0}=\left[\left(\left[a_{0 \lambda}^{n}\right]\right)\right]$ is an element of pro*$H_{P o l}\left(\left(\boldsymbol{S}^{k}, \boldsymbol{s}_{0}\right),\left(\boldsymbol{X}_{0}, \boldsymbol{x}_{0}\right)\right)$ (easily verified) and $k\left(\boldsymbol{a}_{0}\right)=\boldsymbol{a}$, which contradicts the assumption $\boldsymbol{a} \in K$.

Finally, the set $U_{\lambda_{0}}^{a}$ (notation from Lemma 4.5), is open in pro*$\operatorname{HPol}_{0}\left(\left(\mathbf{S}^{k}, \mathbf{s}_{0}\right),\left(\mathbf{X}, \mathbf{x}_{0}\right)\right)$, it contains $\boldsymbol{a}$, and obviously $U_{\lambda_{0}}^{\boldsymbol{a}} \subseteq K$, which completes the proof.

In the previous proof we have used the notation $[f]$ for some pointed homotopy class of a mapping $f$, while in the rest of this paper we only use the notation $f$.

Lemma 4.5. Let $\left(X, x_{0}\right) \in O b\left(S h_{0_{\mathbb{N}}^{*}}^{*}\right)$, let $\boldsymbol{p}:\left(X, x_{0}\right) \rightarrow\left(\boldsymbol{X}, \boldsymbol{x}_{0}\right)=$ $\left(\left(X_{i}, x_{i}\right), p_{i i+1}, \mathbb{N}\right)$ be any sequential HPol Pexpansion of $\left(X, x_{0}\right)$ and let $\boldsymbol{p}^{\prime}$ : $\left(X, x_{0}\right) \rightarrow\left(\boldsymbol{X}^{\prime}, \boldsymbol{x}_{0}^{\prime}\right)=\left(\left(X_{\lambda}^{\prime}, x_{\lambda}^{\prime}\right), p_{\lambda \lambda^{\prime}}^{\prime}, \Lambda\right)$ be any HPol$l_{0}$-expansion of $\left(X, x_{0}\right)$. Let $\boldsymbol{f}=\left[\left(f, f_{\lambda}\right)\right]:\left(\boldsymbol{X}, \boldsymbol{x}_{0}\right) \rightarrow\left(\boldsymbol{X}^{\prime}, \boldsymbol{x}_{0}^{\prime}\right)$ be the canonical isomorphism between those expansions. Then the collection

$$
\mathcal{U}=\left\{U_{\lambda^{\prime}}^{\left[\left(a_{\lambda}^{n}\right)\right]}:\left[\left(a_{\lambda}^{n}\right)\right] \in \operatorname{pro}^{*}-H \operatorname{Pol}_{0}\left(\left(\boldsymbol{S}^{k}, \boldsymbol{s}_{0}\right),\left(\boldsymbol{X}^{\prime}, \boldsymbol{x}_{0}^{\prime}\right)\right), \lambda^{\prime} \in \Lambda\right\},
$$

where

$$
\begin{aligned}
U_{\lambda^{\prime}}^{\left[\left(a_{\lambda}^{n}\right)\right]}= & \left\{\left[\left(b_{\lambda}^{n}\right)\right] \in \text { pro }^{*}-H \operatorname{Pol}_{0}\left(\left(\boldsymbol{S}^{k}, \boldsymbol{s}_{0}\right),\left(\boldsymbol{X}^{\prime}, \boldsymbol{x}_{0}^{\prime}\right)\right):\right. \\
& \left.b_{\lambda^{\prime}}^{n}=a_{\lambda^{\prime}}^{n} \text { for almost all } n \in \mathbb{N}\right\},
\end{aligned}
$$

satisfies properties of topological basis of pro* $-H^{-} \operatorname{Pol}_{0}\left(\left(\boldsymbol{S}^{k}, \boldsymbol{s}_{0}\right),\left(\boldsymbol{X}^{\prime}, \boldsymbol{x}_{0}^{\prime}\right)\right)$, and therefore it generates a unique topology denoted by $\mathcal{T}$. Further, the function

$$
\begin{gathered}
h:\left(\left(\boldsymbol{X}, \boldsymbol{x}_{0}\right)^{\left(\boldsymbol{S}^{k}, \boldsymbol{s}_{0}\right) *}, d^{*}\right) \rightarrow\left(\operatorname{pro}^{*}-\operatorname{HPol}_{0}\left(\left(\boldsymbol{S}^{k}, \boldsymbol{s}_{0}\right),\left(\boldsymbol{X}^{\prime}, \boldsymbol{x}_{0}^{\prime}\right), \mathcal{T}\right),\right. \\
h\left(\left[\left(a_{i}^{n}\right)\right]=\boldsymbol{J}(\boldsymbol{f}) \circ\left[\left(a_{i}^{n}\right)\right]\right.
\end{gathered}
$$

is a homeomorphism.

ProOF. First notice that, for every $\left[\left(c_{\lambda}^{n}\right)\right] \in U_{\lambda^{\prime}}^{\left[\left(a_{\lambda}^{n}\right)\right]}$, it holds $U_{\lambda^{\prime}}^{\left[\left(a_{\lambda}^{n}\right)\right]}=$ $U_{\lambda^{\prime}}^{\left[\left(c_{\lambda}^{n}\right)\right]}$. Now for $\left[\left(c_{\lambda}^{n}\right)\right] \in U_{\lambda_{1}}^{\left[\left(a_{1 \lambda}^{n}\right)\right]} \cap U_{\lambda_{2}}^{\left[\left(a_{2 \lambda}^{n}\right)\right]}$, we have $\left[\left(c_{\lambda}^{n}\right)\right] \in U_{\lambda_{1}}^{\left[\left(c_{\lambda}^{n}\right)\right]} \cap U_{\lambda_{2}}^{\left[\left(c_{\lambda}^{n}\right)\right]}=$ $U_{\lambda_{1}}^{\left[\left(a_{1 \lambda}^{n}\right)\right]} \cap U_{\lambda_{2}}^{\left[\left(a_{2 \lambda}^{n}\right)\right]}$. It follows $\left[\left(c_{\lambda}^{n}\right)\right] \in U_{\lambda_{0}}^{\left[\left(c_{\lambda}^{n}\right)\right]} \subseteq U_{\lambda_{1}}^{\left[\left(c_{\lambda}^{n}\right)\right]} \cap U_{\lambda_{2}}^{\left[\left(c_{\lambda}^{n}\right)\right]}$ for any $\lambda_{0} \geq \lambda_{1}, \lambda_{2}$. Since $\mathcal{U}$ is obviously a cover of pro $^{*}-H \operatorname{Pol}_{0}\left(\left(\boldsymbol{S}^{k}, \boldsymbol{s}_{0}\right),\left(\boldsymbol{X}^{\prime}, \boldsymbol{x}_{0}^{\prime}\right)\right)$ it 
satisfies properties of topological basis. Further, it is obvious that the function $h$ is a bijection. Namely, the function

$$
\begin{gathered}
g:\left(\operatorname{pro}^{*}-H \operatorname{Pol}_{0}\left(\left(\boldsymbol{S}^{k}, \boldsymbol{s}_{0}\right),\left(\boldsymbol{X}^{\prime}, \boldsymbol{x}_{0}^{\prime}\right), \mathcal{T}\right) \rightarrow\left(\left(\boldsymbol{X}, \boldsymbol{x}_{0}\right)^{\left(\boldsymbol{S}^{k}, \boldsymbol{s}_{0}\right) *}, d^{*}\right),\right. \\
g\left(\left[\left(a_{\lambda}^{n}\right)\right]\right)=\boldsymbol{J}\left(\boldsymbol{f}^{-1}\right) \circ\left[\left(a_{\lambda}^{n}\right)\right]
\end{gathered}
$$

is the inverse of $h$. Further, if we take any $\left[\left(a_{i}^{n}\right)\right] \in\left(\left(\boldsymbol{X}, \boldsymbol{x}_{0}\right)^{\left(\boldsymbol{S}^{k}, \boldsymbol{s}_{0}\right) *}, d^{*}\right)$ and any $\lambda^{\prime} \in \Lambda$, using Proposition 3.1, we can check that $h\left(B_{d^{*}}\left(\left[\left(a_{i}^{n}\right)\right], \frac{1}{f\left(\lambda^{\prime}\right)}\right)\right) \subseteq$ $U_{\lambda^{\prime}}^{h\left(\left[\left(a_{i}^{n}\right)\right]\right)}$. Therefore, the continuity of $h$ follows. The continuity of $g$ can be checked analogously.

Proposition 4.6. Let $\left(X_{0}, x_{0}\right)$ be a subspace of $\left(X, x_{0}\right)$, where $\left(X_{0}, x_{0}\right)$, $\left(X, x_{0}\right) \in O b\left(S h_{0_{\mathbb{N}}^{*}}^{*}\right)$, let $r:\left(X, x_{0}\right) \rightarrow\left(X_{0}, x_{0}\right)$ be a retraction and $i:\left(X_{0}, x_{0}\right) \hookrightarrow\left(X, x_{0}\right)$ the inclusion. Then $i_{\#}^{*}:=\check{\pi}_{k}^{* d^{*}}\left(S^{*}\left(H_{0}(i)\right)\right)$ : $\check{\pi}_{k}^{* d^{*}}\left(X_{0}, x_{0}\right) \rightarrow \check{\pi}_{k}^{* d^{*}}\left(X, x_{0}\right)$, where $H_{0}$ is the pointed homotopy functor, is an injective homomorphism of groups and a topological embedding.

Proof. Let $r_{\#}^{*}:=\check{\pi}_{k}^{* d^{*}}\left(S^{*}\left(H_{0}(r)\right)\right): \check{\pi}_{k}^{* d^{*}}\left(X, x_{0}\right) \rightarrow \check{\pi}_{k}^{* d^{*}}\left(X_{0}, x_{0}\right)$ and $G=\operatorname{Im}\left(i_{\#}^{*}\right)$. It is obvious that $i_{\#}^{*}$ and $\left.r_{\#}^{*}\right|_{G}$ are continuous homomorphisms. By showing that $i_{\#}^{*}$ and $\left.r_{\#}^{*}\right|_{G}$ are mutual inverses we will end this proof. Let $A^{*} \in \check{\pi}_{k}^{* d^{*}}\left(X_{0}, x_{0}\right)$. Then

$$
\begin{aligned}
\left(\left.r_{\#}^{*}\right|_{G} \circ i_{\#}^{*}\right)\left(A^{*}\right) & =\left(r_{\#}^{*} \circ i_{\#}^{*}\right)\left(A^{*}\right)=\left(S^{*}\left(H_{0}(r)\right) \circ S^{*}\left(H_{0}(i)\right)\right) \circ A^{*} \\
& =\left(S^{*}\left(H_{0}(r \circ i)\right)\right) \circ\left(A^{*}\right)=1_{\left(X_{0}, x_{0}\right)}^{*} \circ A^{*}=A^{*} .
\end{aligned}
$$

Hereby $1_{\left(X_{0}, x_{0}\right)}^{*}$ denotes the identity of $S h_{0_{\mathbb{N}}}^{*}$, and we used $r \circ i=i d$ and the functorial properties. This also implies that $i_{\#}^{*}$ is injective.

Now let $B^{*} \in G$. Then there is a unique $B_{0}^{*} \in \check{\pi}_{k}^{* d^{*}}\left(X_{0}, x_{0}\right)$ such that $B^{*}=i_{\#}^{*}\left(B_{0}^{*}\right)$. Therefore

$\left(\left.i_{\#}^{*} \circ r_{\#}^{*}\right|_{G}\right)\left(B^{*}\right)=\left(\left.i_{\#}^{*} \circ r_{\#}^{*}\right|_{G}\right)\left(i_{\#}^{*}\left(B_{0}^{*}\right)\right)=i_{\#}^{*}\left(\left(\left.r_{\#}^{*}\right|_{G} \circ i_{\#}^{*}\right)\left(B_{0}^{*}\right)\right)=i_{\#}^{*}\left(B_{0}^{*}\right)=B^{*}$, where in the third equality we used the first part of the proof.

Corollary 4.7. Let $X$ be a compact metric space, $X_{0} \subseteq X$ its retract and $x_{0} \in X_{0} \subseteq X$ any point. Then $\check{\pi}_{k}^{* d^{*}}\left(X_{0}, x_{0}\right)$ can be considered as a closed topological subgroup of $\check{\pi}_{k}^{* d^{*}}\left(X, x_{0}\right)$.

Proof. This follows from Propositions 4.4, 4.6 and the following facts: $X_{0}$ is closed in $X$ as a retract of Hausdorff space; $X_{0}$ is a compact metric space; every closed subspace of paracompact space $X$ is normally embedded in $X$.

Thus, not only that we can consider $\check{\pi}_{k}^{* d^{*}}\left(X_{0}, x_{0}\right)$ as a topological subgroup of $\check{\pi}_{k}^{* d^{*}}\left(X, x_{0}\right)$, provided $X_{0}$ is a retract of compact metric space $X$, 
but also the "closeness" property of $X_{0}$ in $X$ is translated to relation between topological coarse shape groups.

Now let us recall the notion of coarse shape path connectedness (see more in [3]) and show that $\check{\pi}_{k}^{* d^{*}}\left(X, x_{0}\right)$ do not depend on $x_{0} \in X$.

A coarse shape path in a topological space $X$ from $x_{0}$ to $x_{1}, x_{0}, x_{1} \in$ $X$, is every morphism $\Omega^{*}:(I, 0,1) \rightarrow\left(X, x_{0}, x_{1}\right)$ of the category $S h_{00}^{*} \equiv$ $S h_{\left(H T{ }_{0} p_{00}, H P_{o l} l_{00}\right)}$. A space $X$ is said to be coarse shape path connected if for every two points $x_{0}, x_{1} \in X$ there is a coarse shape path in $X$ from $x_{0}$ to $x_{1}$. It is known that the path connectedness implies shape path connectedness, which further implies the coarse shape path connectedness.

Proposition 4.8. Let $\Omega^{*}:(I, 0,1) \rightarrow\left(X, x_{0}, x_{1}\right)$ be a coarse shape path from $x_{0}$ to $x_{1}$ in a space $X$, where $\left(X, x_{0}, x_{1}\right)$ admits a sequential $H_{P o l_{00}-}$ expansion. Let $\boldsymbol{p}:\left(X, x_{0}, x_{1}\right) \rightarrow\left(\boldsymbol{X}, \boldsymbol{x}_{0}, \boldsymbol{x}_{1}\right)=\left(\left(X_{i}, x_{i}, x_{i^{\prime}}\right), p_{i i+1}, \mathbb{N}\right)$ be an $H$ Pol $_{00}$-expansion of $\left(X, x_{0}, x_{1}\right)$ and let $\left[\left(\omega_{i}^{n}\right)\right]:(I, 0,1) \rightarrow\left(\boldsymbol{X}, \boldsymbol{x}_{0}, \boldsymbol{x}_{1}\right)$ represent $\Omega^{*}$ in pro $^{*}-H \operatorname{Pol}_{00}\left((I, 0,1),\left(\boldsymbol{X}, \boldsymbol{x}_{0}, \boldsymbol{x}_{1}\right)\right)$. Then $i_{\Omega^{*}}: \check{\pi}_{k}^{* d^{*}}\left(X, x_{0}\right) \rightarrow$ $\check{\pi}_{k}^{*} d^{*}\left(X, x_{1}\right)$,

$$
i_{\Omega^{*}}\left(A^{*}=\left\langle\left[\left(a_{i}^{n}\right)\right]\right\rangle\right)=\left\langle\left[\left(i_{\omega_{i}^{n}}\left(a_{i}^{n}\right)\right)\right]\right\rangle,
$$

is an isomorphism of topological groups, for every $k \in \mathbb{N}$, where $i_{\omega_{i}^{n}}$ : $\pi_{k}\left(X_{i}, x_{i}\right) \rightarrow \pi_{k}\left(X_{i}, x_{i}^{\prime}\right)$ is the isomorphism of homotopy groups induced by $\omega_{i}^{n}$.

REMARK 4.9. There are $H$ Pol $_{0}$-expansions of $\left(X, x_{0}\right)$ and $\left(X, x_{1}\right)$ that are induced by $\boldsymbol{p}$ (by forgetting base points $x_{1}, x_{0}$, respectively).

Proof. It is well known that $i_{\Omega^{*}}$ is an isomorphism of groups having the inverse $\left(i_{\Omega^{*}}\right)^{-1}=i_{\left\langle\left[\left(\bar{\omega}_{i}^{n}\right)\right]\right\rangle}$, where $\bar{\omega}_{i}^{n}$ is the inverse path (precisely the homotopy class of inverse path) of $\omega_{i}^{n}$, for every $i, n \in \mathbb{N}$. We have to prove the continuity of $i_{\Omega^{*}}$ and $\left(i_{\Omega^{*}}\right)^{-1}$ only. Let $m \in \mathbb{N}$ and $A^{*} \in \check{\pi}_{k}^{* d^{*}}\left(X, x_{0}\right)$. Then, by the fact that $i_{\omega_{i}^{n}}: \pi_{k}\left(X_{i}, x_{i}\right) \rightarrow \pi_{k}\left(X_{i}, x_{i}^{\prime}\right)$ is an isomorphism, for every $i, n \in \mathbb{N}$, we can easily see that $i_{\Omega^{*}}\left(B_{d^{*}}\left(A^{*}, 1 / m\right) \subseteq B_{d^{*}}\left(i_{\Omega^{*}}\left(A^{*}\right), 1 / m\right)\right.$, so continuity of $i_{\Omega^{*}}$ follows. The continuity of $\left(i_{\Omega^{*}}\right)^{-1}$ can be proven analogously.

COROLlary 4.10. If $X$ is a coarse shape path connected topological space admitting a sequential HPol $0_{0}$-expansion, then $\check{\pi}_{k}^{* d^{*}}\left(X, x_{0}\right) \simeq \check{\pi}_{k}^{* d^{*}}\left(X, x_{1}\right)$, for any choice of $x_{0}, x_{1} \in X$.

Corollary 4.11. If $X$ is a metrizable continuum, then $\check{\pi}_{k}^{*} d^{*}\left(X, x_{0}\right)$ does not depend on $x_{0} \in X$.

Proof. Every connected compact metric space is coarse shape path connected.

At the end we provide few interesting examples. 
EXAMPLE 4.12. If $X$ is a stable space (a space which admits a rudimentary expansion) then $\check{\pi}_{k}^{*} d^{*}\left(X, x_{0}\right)$ is discrete for every $k \in \mathbb{N}$ and $x_{0} \in X$. Namely, it is obvious that if we take an $H$ Pol $_{0}$-expansion $\boldsymbol{p}:\left(X, x_{0}\right) \rightarrow$ $\left(\left(Y, y_{0}\right)\right)$ of $\left(X, x_{0}\right)$, where $\left(\left(Y, y_{0}\right)\right)$ is a rudimentary inverse system in $\mathrm{HPol}_{0}$ containing only one object $\left(Y, y_{0}\right)$, then the ball $B_{d^{*}}\left(A^{*}, 1\right)=\left\{A^{*}\right\}$ is the singleton, for every $A^{*}=\left\langle\left[\left(a^{n}\right)\right]\right\rangle \in \check{\pi}_{k}^{* d^{*}}\left(X, x_{0}\right)$. Let us recall that polyhedra are stable spaces. Also, classical example of a stable space which is not polyhedron is the Warsaw circle.

Example 4.13. Let $X$ be the Hawaiian earring. Then, by Example 4.5. of [5] we have that $\check{\pi}_{1}^{d}(X)$ is not discrete. Therefore, by using Proposition 4.2 we infer that $\check{\pi}_{1}^{*} d^{*}(X)$ is not discrete (we are not specifying a base point since $X$ is connected compact metric space). Also, there is an uncountable discrete closed topological subgroup of $\check{\pi}_{1}^{* d^{*}}(X)$. Namely, the 1-dimensional sphere $S^{1}$ (also any finite wedge of 1-dimensional spheres) is a retract of $X$, so the conclusion follows by Corollary 4.7, previous example and well known fact that $\check{\pi}_{1}^{*}\left(S^{1}, s_{0}\right)=\left(\prod_{n \in \mathbb{N}} \mathbb{Z}\right) /\left(\bigoplus_{n \in \mathbb{N}}^{\mathbb{Z}}\right)$.

EXAMPLE 4.14. We give an example of a space which is not stable, but still has a (nontrivial) discrete topological coarse shape group at every point. Let $(D, \star)$ be the dyadic solenoid, meaning that $(D, \star)=\lim _{\leftarrow}$ $\left(\left(X_{i}, x_{i}\right), p_{i i+1}, \mathbb{N}\right)$ taken in Top $_{0}$, where $X_{i}=S^{1}=\{z \in \mathbb{C}:|z|=1\}$ and $p_{i i+1}(z)=z^{2}$, for every $i \in \mathbb{N}$. It is known that $\check{\pi}_{1}(D, \star)$ is trivial, so $\check{\pi}_{1}^{d}(D, \star)$ is trivial discrete. Also, by Example 2. from [1] we know that $\check{\pi}_{1}^{*}(D, \star)$ is uncountable and every element of that group can be represented by a pro* $^{*}$ $\mathrm{HPol}_{0}$ morphism $\left[\left(\left[a_{i}^{n}\right]\right)\right]:\left(S^{1}, s_{0}\right) \rightarrow\left(\left(X_{i}, x_{i}\right),\left[p_{i i+1}\right], \mathbb{N}\right)$,

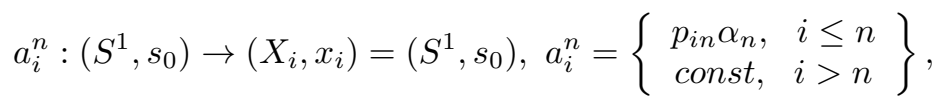

where $\alpha=\left(\left[\alpha_{k}\right]\right)$ is a sequence of pointed homotopy maps $\left[\alpha_{k}\right]:\left(S^{1}, s_{0}\right) \rightarrow$ $\left(S^{1}, s_{0}\right)$, which can be considered as a sequence in $\mathbb{Z}=\pi_{1}\left(S^{1}\right)$. Also, we know that $\alpha=\alpha^{\prime}$ almost everywhere is equivalent to $\left[\left(\left[a_{i}^{n}\right]\right)\right]=\left[\left(\left[a_{i}^{\prime n}\right]\right)\right]$, where $\left[\left(\left[a_{i}^{n}\right]\right)\right],\left[\left(\left[a_{i}^{\prime n}\right]\right)\right]$ are generated by $\alpha, \alpha^{\prime}$ with respect to the previous relations (see [2]). We will prove that $\check{\pi}_{1}^{*} d^{*}(D, \star)$ is discrete by proving that a distance between two different points is always 1 . Let us assume to the contrary that there are two different points $A^{*}=\left\langle\left[\left(\left[a_{i}^{n}\right]\right)\right]\right\rangle, A^{\prime *}=\left\langle\left[\left(\left[a_{i}^{\prime n}\right]\right)\right]\right\rangle \in \check{\pi}_{1}^{*} d^{*}(D, \star)$ such that $d^{*}\left(\left[\left(\left[a_{i}^{n}\right]\right)\right],\left[\left(\left[a_{i}^{\prime n}\right]\right)\right]\right)<1$ (because the distance is always less or equal $1)$. By the definition of $d^{*}$, that means $d^{*}\left(\left[\left(\left[a_{i}^{n}\right]\right)\right],\left[\left(\left[a_{i}^{\prime n}\right]\right)\right]\right) \leq 1 / 2$, which implies that $\left[p_{1 n} \alpha_{n}\right]=\left[p_{1 n} \alpha_{n}^{\prime}\right]$ for almost all $n \in \mathbb{N}$, where the sequences $\alpha=\left(\left[\alpha_{k}\right]\right)$ and $\alpha^{\prime}=\left(\left[\alpha_{k}^{\prime}\right]\right)$ generate $\left[\left(\left[a_{i}^{n}\right]\right)\right]$ and $\left[\left(\left[a_{i}^{\prime n}\right]\right)\right]$, respectively. Since, $p_{1 n}(z)=z^{2^{n-1}}$, and $\pi_{1}\left(\left[p_{1 n}\right]\right): \mathbb{Z} \rightarrow \mathbb{Z}, \pi_{1}\left(\left[p_{1 n}\right]\right)\left(\left[\alpha_{n}\right]\right)=\left[p_{1 n} \alpha_{n}\right]$ is actually the multiplication by $2^{n-1}$, it follows that $\pi_{1}\left(\left[p_{1 n}\right]\right)$ is a monomorphism and 
we infer that $\left[\alpha_{k}\right]=\left[\alpha_{k}^{\prime}\right]$ for almost all $k \in N$. Therefore $\left[\left(\left[a_{i}^{n}\right]\right)\right]=\left[\left(\left[a_{i}^{\prime n}\right]\right)\right]$ i.e. $A^{*}=A^{\prime *}$, which is a contradiction.

\section{REFERENCES}

[1] N. Koceić Bilan, The coarse shape groups, Topology Appl. 157 (2010) 894-901.

[2] N. Koceić Bilan, Computing coarse shape groups of solenoids, Math. Commun. 14 (2014), 243-251.

[3] N. Koceić Bilan and N. Uglešić, The coarse shape path connectedness, Glas. Mat. Ser. III 46(66) (2011), 491-505.

[4] N. Koceić Bilan and N. Uglešić, The coarse shape, Glas. Mat. Ser. III 42(62) (2007), $145-187$.

[5] T. Nasri, F. Ghanei, B. Mashayekhy and H. Mirebrahimi, On topological shape homotopy groups, Topology Appl. 198 (2016), 22-33.

[6] F. Ghanei, H. Mirebrahimi, B. Mashayekhy and T. Nasri, Tayyebe, Topological coarse shape homotopy groups, Topology Appl. 219 (2017), 17-28.

[7] S. Mardešić and J. Segal, Shape theory, North-Holland, Amsterdam, 1982.

[8] N. Uglešić, Metrization of pro-morphism sets, Glas. Mat. Ser. III 44(64) (2009), 499531.

[9] N. Uglešić, Ultrametrization of pro*-morphism sets, Math. Commun. 18 (2013), 19-47.

\section{Topološke grupe gruboga oblika kompaktnih metričkih prostora}

\section{Nikola Koceić Bilan i Zdravko Čuka}

SAžEtaK. Teorija oblika i, relativno nova, teorija gruboga oblika su se pokazale vrlo korisnima u proučavanju topoloških prostora kao i odgovarajućih algebarskih invarijanti, posebno, grupa oblika i gruboga oblika. Primjenom pogodnih ultrametrika na posebne skupove pro i pro*$^{*}$-morfizama topologiziramo grupe (gruboga) oblika kompaktnih metričkih prostora tako da one postaju topološke grupe. Kod oblika, ove topološke grupe su izomorfne nedavno konstruiranim topološkim homotopskim grupama oblika, dok kod gruboga oblika dobivamo nove invarijante koje označujemo sa $\check{\pi}_{k}^{* d^{*}}\left(X, x_{0}\right)$. Dokazujemo i neka važna svojstva grupa $\check{\pi}_{k}^{* d^{*}}\left(X, x_{0}\right)$ i navodimo nekoliko zanimljivih primjera.

Nikola Koceić Bilan

Faculty of science

University of Split

Ruđera Boškovića 33, 21000 Split, Croatia

E-mail: koceic@pmfst.hr

Zdravko Čuka

Faculty of civil engineering, architecture and geodesy

University of Split

21000 Split, Croatia

Received: 4.3.2017. 